\title{
IMPORTANCIA DE LA PRODUCCIÓN DE LA CONCHA PRIETA (Anadara tuberculosa) EN LAS COSTAS ECUATORIANAS
}

\section{THE IMPORTANCE OF THE PRODUCTION OF BLACK SHELL (Anadara tuberculosa) ON THE ECUADORIAN COAST}

\author{
Eveligh Prado-Carpio ${ }^{1}$, Moisés Enrique Martínez-Soto ${ }^{2}$, Anne Morris Díaz ${ }^{3}$, Cristhian Castro-Armijos ${ }^{4}$, Patricio Renteria- \\ Minuche ${ }^{1}$, Julián Coronel-Reyes ${ }^{4}$, Carlos Rodríguez Monroy ${ }^{5}$ \\ ${ }^{1}$ Universidad Técnica de Machala, Ecuador, CP 070102 \\ ${ }^{2}$ Universidad Simón Bolívar, Colombia, CP 4001 \\ ${ }^{3}$ Universidad del Zulia / INSTGECON, Venezuela, CP 4001 \\ ${ }^{4}$ Centro de especialización y capacitación agropecuaria CECAGROP, CP070102 \\ ${ }^{5}$ Universidad Politécnica de Madrid, España, CP 28006 \\ Email: eprado@utmachala.edu.ec
}

\section{Información del artículo}

Tipo de artículo: Artículo de revisión

Recibido:

$27 / 03 / 2020$

Aceptado:

$17 / 06 / 2020$

Licencia: CC BY-NC-SA 4.0

Revista

ESPAMCIENCIA

11(1):34-46

DOI:

https://doi.org/10.51

260/revista_espamci encia.v11i1.209

\section{Resumen}

La extracción de la concha prieta (Anadara tuberculosa) es una actividad productiva ancestral, que se realiza de manera espontánea e intuitiva y cuya importancia no ha sido suficientemente ponderada, destacada y reconocida en los diversos ámbitos gubernamentales, empresariales y académicos del Ecuador y de otros países que también la producen. Esta investigación tiene por objetivo indagar sobre los indicadores que determinan la importancia económica, social y ambiental a nivel global y nacional de la producción del molusco bivalvo concha prieta. A tal fin, se aplicó una metodología de investigación bibliográfica de fuentes primarias, secundarias y terciarias. Se obtuvo como resultado que su aporte al PIB ecuatoriano es de 15 millones de USD al año, si se incluye el valor que se agrega a la concha prieta a nivel de restaurante, picanterías y cevicherías, que genera unos 8000 puestos de trabajo de tiempo completo y parcial, la mayoría por cuenta propia. Contribuye a preservar más de 156 mil hectáreas de superficie de manglar existentes todavía en las costas ecuatorianas. Se concluye que la producción de la concha prieta registra una relevancia significativa en términos económicos, sociales y ambientales, con un gran potencial para insertarse de manera formal en el circuito exportador ecuatoriano, de mejorar los niveles de ingresos y calidad de vida de los concheros y de contribuir al manejo sostenible del ecosistema manglar. Se recomienda profundizar en el estudio de las variables económicas, sociales y ambientales que tienen mayor incidencia sobre sus niveles de sostenibilidad y competitividad.

Palabras clave: Importancia económica, importancia social, importancia ambiental, molusco bivalvo

\begin{abstract}
The collection of black shells (Anadara tuberculosa) is an ancestral productive activity, which is carried out spontaneously and intuitively and whose importance has not been sufficiently considered, highlighted and recognized in the various governmental, business and academic spheres in Ecuador and other countries. The objective of this research is to find the indicators that determine the economic, social and environmental importance of the production of this bivalve mollusc, at a global and national level. To this end, a methodology of bibliographical research of primary, secondary and tertiary sources was applied. The contribution of black shell collection to Ecuador's GDP is US\$ 15 million per year, if one includes the value added to the concha prieta at the restaurant level, which generates some 8,000 full-time and part-time jobs, mostly self-employment. It contributes to the preservation of more than 156,000 hectares of mangrove area still existing on the Ecuadorian coast. It is concluded that the production of black shells is of significant relevance in economic, social and environmental terms, with a great potential to be formally inserted in the Ecuadorian export circuit, to improve the levels of income and quality of life of the concheros (shell collectors) and to contribute to the sustainable management of the mangrove ecosystem. It is recommended to deepen the study of the economic, social and environmental variables that have greater impact on their levels of sustainability and competitiveness.
\end{abstract}

Keywords: Economic importance, social importance, environmental importance, bivalve mollusk. 


\section{INTRODUCCIÓN}

La alimentación y la agricultura son fundamentales para la consecución de todos los objetivos de desarrollo sostenible (ODS), y en especial el número 14, que hace énfasis en conservar y utilizar sosteniblemente los océanos, los mares y los recursos marinos para el desarrollo sostenible (FAO, 2018) y la alimentación de la población.

Según Mora et al. (2010), la concha prieta cuyo nombre científico es (Anadara tuberculosa) (Sowerby, 1833) (Arcidae) es un recurso marino del Ecuador, la misma es un molusco bivalvo que se produce naturalmente en los manglares de la Costa ecuatoriana y de otros países de la costa del Pacífico americano, desde el sur de México hasta el norte del Perú (Silva-Benavides y Bonilla, 2014).

La extracción de este bivalvo es una actividad productiva de la cual dependen, para obtener su sustento, diario miles de familias en las costas del Ecuador y del Pacífico ecuatorial americano (Prado-Carpio et al., 2018a).

Sin embargo, existen múltiples problemas y amenazas, que han sido estudiados desde el punto de vista biológicoecológico (Borda y Cruz, 2004; Diringer et al, 2019; Zavala-Arellano, 2019; González-Banchón y SolanoVera, 2019; Murillo-Rivas, 2018 y Panta-Vélez et al, 2020), desde el punto de vista social (Caicedo, 2014; Beitl, 2010; Dazzini-Langdon, 2020; Mendoza-Portillo, et al, 2020 y Calispa-Quinto, 2018) o desde el punto de vista económico (Cabanilla 2007 y 2010; Tapia-Santos, 2020; Dioses-Huayna, 2019; Ortiz-Bolaños, 2019). Pero, son pocos los estudios realizados con un enfoque que integren lo ambiental, lo social y lo económico, es decir un enfoque de producción sostenible.

La concha prieta es un recurso económico (Lucero et al., 2012) que puede alcanzar una posición significativa en el modelo productivo y de exportación ecuatoriano (RendónYllescas et al., 2009), al tiempo de servir de medio de vida para 3000 familias (MAGAP, 2014).

La producción de la Anadara tuberculosa es una actividad productiva ancestral (Prado-Carpio et al., 2018b; PachecoNavarro, 2017; Arias-Morán y Molina, 2017; Antón y Fierro, 2019 y Morocho et al., 2019), que se realiza de manera espontánea e intuitiva, cuya importancia no ha sido suficientemente ponderada, destacada y reconocida en diversos ámbitos gubernamentales, sociales y académicos del Ecuador y de otros países que también la producen.

Por tanto, esta investigación tiene por objetivo indagar sobre los indicadores que evidencian la importancia económica, social y ambiental de la producción de la concha prieta (Anadara tuberculosa) a nivel global y en el Ecuador.

\section{MATERIALES Y MÉTODOS}

La metodología aplicada fue la de la investigación bibliográfica de fuentes primarias, secundarias y terciaras (Cortes-Osorio, 2020; Molar-Orozco, 2020; CamposOcampo, 2017).

Según Gómez-Luna et al. (2014) la metodología propuesta para la revisión bibliográfica puede ser aplicada a cualquier tema de investigación para determinar la relevancia e importancia del mismo y asegurar la originalidad de una investigación. Además, permite que otros investigadores consulten las fuentes bibliográficas citadas, pudiendo entender y quizá continuar el trabajo realizado. La metodología propuesta se compone de cuatro fases: definición del problema, búsqueda de la información, organización de la información y análisis de la información.

\section{RESULTADOS Y DISCUSIÓN}

En este apartado, se presentan y analizan los resultados de la información relativa a la importancia de la producción de la concha prieta (Anadara tuberculosa), lo cual se pone de manifiesto a través de variables e indicadores de tipo económico, social y ambiental (Figura 1)

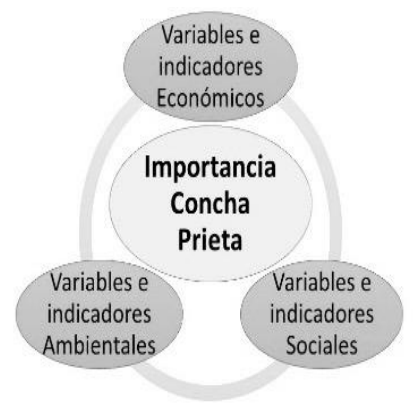

Figura 1. Esquema de variables e indicadores para el análisis de la importancia de la concha prieta en el Ecuador.

En la figura 1 se observa como la importancia de la concha prieta se encuentra en el centro de una dinámica productiva caracterizada por variables e indicadores de tipo económico, ambiental y social, los cuales se presentan y analizan a continuación.

Importancia económica de la producción de los moluscos bivalvos y de la concha prieta.

Según FAO (2018), los moluscos bivalvos representaron un $6 \%$ del comercio mundial de los pescados y productos pesqueros en el año 2016, luego de los pescados y los crustáceos. Las especies de bivalvos más comercializadas son los mejillones, las almejas, los peines y las ostras, siendo el arca del Pacífico Occidental cuyo nombre 
científico es Anadara granosa, la única del mismo género que la concha prieta, que evidencia jerarquía en el comercio internacional de moluscos bivalvos con un $3 \%$ del total (FAO, 2018), ubicándose entre las 10 especies de bivalvos más comercializadas internacionalmente.

La gran mayoría de estos bivalvos de importancia en el comercio internacional son cultivados (Cáceres-Martínez y Vásquez-Yeomans, 2014), es decir, no se producen como resultado de la captura de especímenes de poblaciones naturales como es el caso de la concha prieta en Ecuador (Mora y Moreno, 2009) y otros países del Pacífico Oriental, sino a través de diversos sistemas de producción acuícolas. En este sentido, para el año 2016, la producción mundial de moluscos producidos en acuicultura, alanzó la cifra de 17139 millones de TM de peso vivo, un $21,41 \%$ de la producción acuícola mundial que fue de 80131 millones de TM de peso vivo (FAO, 2018).

China es con ventaja el mayor exportador de bivalvos (Wijsman et al., 2019), pues en 2016 sus exportaciones fueron tres veces superiores a las de Chile, el segundo mayor exportador. China también posee un consumo nacional importante, aunque la Unión Europea es el mayor mercado importador de bivalvos (FAO, 2018).

El consumo de bivalvos se promueve ampliamente por ser considerados productos alimentarios saludables y sostenibles, por tanto, su demanda ha ido aumentando en los últimos años (Lee et al., 2010; Cañas-García y Sierra, 2019).

Los moluscos bivalvos son animales filtradores (PintóSolé et al., 2011; Ordinola et al., 2019; Tripaldi, et al., 2016; Rivas-Mejía, 2018) y requieren un mínimo de manejo y cuidado, no necesitando más alimento que las algas presentes en su hábitat natural (Farías, 2008).

Su extracción se ha realizado durante muchos siglos, pero ahora, los avances tecnológicos en el campo de la acuicultura han permitido incrementar los niveles de producción significativamente, lo cual contribuye a satisfacer la demanda creciente (IAES, S/F).

Según Helm et al. (2006) esta situación se evidencia en la evolución de la producción mundial de este rubro, que a finales del siglo XX alcanzó la cifra de 14,2 millones de $\mathrm{TM}$, de los cuales aproximadamente 4,0 millones de TM se originaban en la captura de poblaciones naturales, por tanto, la producción acuícola era mayor a los 10 millones de TM (Figura 2).

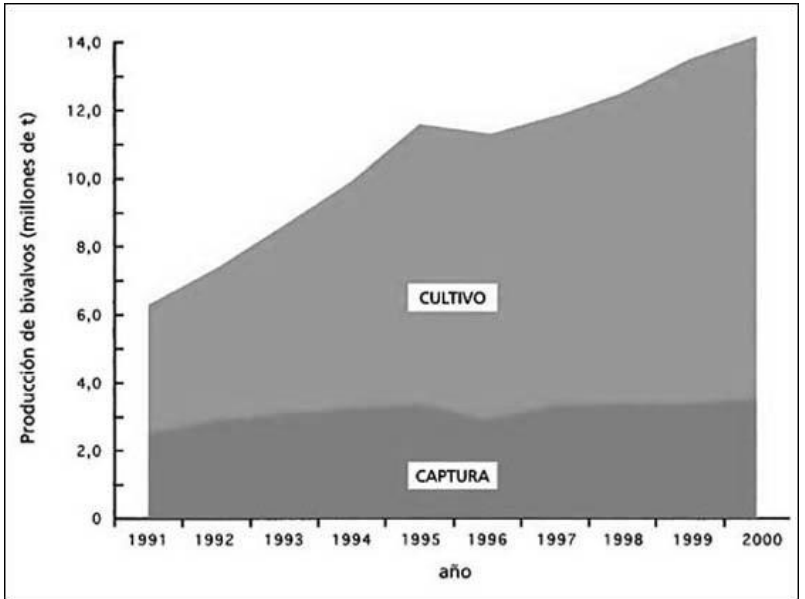

Figura 2. Producción global de bivalvos procedentes de la pesca y de la acuicultura durante el decenio 19912000.

Fuente: Helm et al. (2006)

Según la FAO (2016), en Europa se produjeron 632000 toneladas de bivalvos y sus principales productores fueron España (223 000 toneladas), Francia (155000 toneladas) e Italia (111 000 toneladas) en el año 2014. La producción de bivalvos en China ese mismo año fue de unos 12 millones de toneladas, cinco veces mayor que la del resto del mundo. Otros importantes productores asiáticos de bivalvos son, por ejemplo, el Japón (377 000 toneladas), la República de Corea (347000 toneladas) y Tailandia (210 000 toneladas).

La Unión Europea es uno de los principales mercados de bivalvos y capta más de un tercio del comercio total, pero solo 13 países no comunitarios están autorizados a enviar bivalvos vivos al mercado de esta, lo que deja en evidencia el estricto control sanitario de este tipo de productos pesqueros (Ipac, 2017).

Según el Observatorio de la Complejidad Económica (2018), los principales importadores a nivel mundial son España (\$1,67 Miles de millones), Japón (\$1,35 Miles de millones), Italia ( $\$ 1,28$ Miles de millones), Hong Kong (\$1,1 Miles de millones) y los Estados Unidos (\$971 Millones).

En América Latina, la producción de moluscos bivalvos alcanzó aproximadamente 128500 toneladas lo que representa el $1,07 \%$ del total mundial de la producción acuícola. Es decir, su participación es prácticamente nula en el comercio internacional de los moluscos bivalvos, por tanto, su importancia se circunscribe al consumo local o intraregional (Lovatelli et al., 2008). 
Chile es el mayor productor y exportador en la región seguido por Brasil y Perú, siendo las principales especies producidas el mejillón chileno y la concha de abanico. En los recientes años, las exportaciones totales de bivalvos de América Latina y el Caribe fueron 18500 toneladas (Luchini y Panné-Huidobro, 2008).

Según la Ipac (2017), citando un informe de la Globefish sobre el mercado de bivalvos en el mundo se confirma que a pesar de las importantes cifras de producción, un escaso volumen que se dirige al comercio internacional, algo menos del $5 \%$, esto se debe a su naturaleza de altamente perecederos, y la peligrosidad potencial para la salud humana cuando no se manejan adecuadamente. $Y$ es por ello que la mayor parte de los bivalvos se consumen en los propios países de producción.

Los datos antes presentados permiten inferir la reducida participación de los moluscos bivalvos en el comercio internacional de productos marinos y acuícolas, pero al mismo tiempo su significativa participación en el abastecimiento de mercados nacionales y locales, así como el crecimiento de la producción con técnicas acuícolas.

En el caso de la "concha prieta" o Anadara tuberculosa, su importancia económica se circunscribe geográficamente al Pacifico americano, desde el sur de México, hasta el norte del Perú (Mendoza y Alvitres, 2013), zona en la cual se produce casi totalmente de manera natural.

Su captura en los manglares de la zona marino costera, representando una actividad económica doméstica y de subsistencia para las comunidades de pescadores, quienes encuentran en la extracción, comercialización y preparación de la concha prieta, una fuente de ingresos de carácter local (Lazarich- Gener, 2009).

Además, en el caso particular de Colombia, Ecuador y Perú, se ha desarrollado un comercio internacional de concha prieta (Azabache, 2016 y Espinosa et al., 2010), de carácter informal, del cual no se han encontrado registros estadísticos que cuantifiquen este comercio entre estos países (Mendoza, 2015).

Comparado con otros rubros económicos, no es práctico o viable llevar dichos registros, porque probablemente este tipo de comercio no cumple con las normativas sanitarias y tributarias vigentes en los países indicados o porque no existen normativas específicas que regulen esta actividad comercial.

Sin embargo, según el MAGAP (2014), alrededor del 85\% de estas capturas de Anadara tuberculosa que se realizan en Colombia se destina al mercado ecuatoriano. Asimismo, Ordinola et al. (2019), describe que para abastecer la demanda de Anadara tuberculosa en Perú, se ha llegado a importar conchas de Ecuador y Colombia.

De lo anteriormente planteado se infiere que este comercio de la concha prieta fluye de un país a otro de manera recíproca y alternativa, con base en la dinámica económica de carácter fronterizo, influenciada por la tasa de cambio, por la oferta y demanda interna de la concha prieta y por las características propias de la economía de cada uno de estos países.

En el caso del Ecuador, si se toma máximo de producción registrado de 34,4 millones de conchas en un año (Rendón-Yllescas et al., 2009) y si se realiza un cálculo con base en 10 USD el ciento de conchas a nivel de los pescadores o concheros (Cabanilla, 2010; Fundación de Defensa Ecológica, 2009) o de 17 a 20 USD a nivel de comerciantes detallistas (Cabanilla, 2010; Prado-Carpio et al., 2018b y Valencia-Caicedo, 2013).

Se puede estimar que el aporte al PIB ecuatoriano de la concha prieta se encuentra, entre 3,4 y 6,8 millones de USD. Esta cifra se pudiera incrementar a 10 a 15 millones de USD al año, si se incluye el valor que se agrega a la concha prieta a nivel de restaurantes, picanterías y cevicherías (Prado-Carpio et al., 2018b).

Por tanto, aunque su importancia económica actual es relativamente reducida si es comparada, por ejemplo, con los sectores: camaronero o bananero ecuatoriano, no es menos interesante que la concha prieta presenta un interesante potencial de crecimiento a futuro, con base en el propio consumo nacional y en el comercio internacional (Cabanilla, 2007).

Con países vecinos (Colombia y Perú) e inclusive, hacia localidades en el extranjero, como España, en las cuales las colonias de emigrantes ecuatorianos son numerosas y podrían convertirse en un mercado objetivo (RendónYllescas et al., 2009); así como una posibilidad cierta de alcanzar un abastecimiento nacional, reducir las importaciones y agregar un nuevo rubro a la matriz de exportaciones del modelo económico ecuatoriano, contribuyendo a mejorar su balanza comercial.

\section{Importancia social de la producción de la concha prieta}

A nivel global, según la FAO (2018), aproximadamente 59,6 millones de personas trabajaron (a tiempo completo, tiempo parcial u ocasionalmente) en el sector primario de la pesca de captura y la acuicultura en 2016, de los cuales 19,3 millones lo hicieron en la acuicultura y 40,3 millones en la pesca de captura. Se calcula que alrededor del $14 \%$ de estos trabajadores eran mujeres. En 2016, el 85\% de la población mundial empleada en los sectores de la pesca y 
la acuicultura se encontraba en Asia, seguida de África (10\%) y América Latina y el Caribe (4\%).

En el Ecuador, para el caso de la cadena de producción del agronegocio de la concha prieta, según cifras del Banco Interamericano de Desarrollo (BID, 2017) se estima que unas 3000 personas trabajan como concheros a los cuales hay que sumar unas 500 personas adicionales que laboran como lancheros-transportistas de los concheros, comerciantes, contadores de concha, transportistas de concha y otras labores relacionadas.

A esta cifra de trabajadores debe agregarse la de los cientos de trabajadores de cevicherías, picanterías y restaurantes que sirven concha prieta en cada una de las ciudades y poblaciones de la Costa ecuatoriana, y en todo el país en general. Los cuales, si bien es cierto tienen una oferta de variada de platos a sus comensales, agregan la concha prieta como parte de la mixtura alimenticia de los platos que ofrecen y en otros casos, se sirve también la concha prieta como principal materia prima o ingrediente de los platos preparados, a través de ceviche de concha, arroz con concha, las conchas asadas y otros que forman parte de la gastronomía ecuatoriana (BenalcázarBenavides, 2015).

Es decir, que se podría estimar en unos 8 mil puestos de trabajo, los que, a tiempo completo o tiempo parcial, en mayor o menor grado, están relacionados con el agronegocio de la concha prieta en el Ecuador (PradoCarpio et al., 2018b). La gran mayoría de estos puestos de trabajo con remuneración precaria que no llega al salario mínimo mensual o son desarrollados por emprendedores, micros y pequeños empresarios, así como trabajadores por cuenta propia (Valencia-Caicedo, 2013).

Si bien, el tamaño de la economía de la concha prieta es relativamente pequeño si se compara con el sector camaronero, el cual según Camposano (2020) en el año 2019 se estimó que exportó 1400 millones de libras equivalentes a 3600 millones de dólares (USD).

Se hace necesario destacar que, a nivel microeconómico local, la actividad socioeconómica de la concha prieta tiene gran relevancia para la sustentación de pequeñas comunidades costeras de pescadores (Muñoz-Suarez, 2018; Alemán-Mejía et al., 2019), a lo largo de su costa pacífica, y principalmente en las provincias de Esmeraldas, Guayas, Manabí y de El Oro (RendónYllescas et al., 2009), en las cuales son extraídas (conchadas).

La misma es fuente de puestos de trabajo, ingresos monetarios estables a las familias, alimentos para autoconsumo (Gómez, 2011), así como generadora de gran riqueza cultural, artística y culinaria de la población ecuatoriana, principalmente, la que habita en la costa pacífica, la cual es la mayoría (Alcívar-Bravo y PeñafielRivera, 2018).

Un elemento a resaltar en la importancia social de la concha prieta, consiste en su valor nutricional (Cruz et al. 2012), por lo tanto, se puede señalar que los principales beneficiaros de su producción son los consumidores de los centros urbanos y rurales, que tienen su preferencia alimenticia por la concha prieta, ya sea fresca o procesada, sola o acompañada, en virtud de su sabor singular y sus excelentes características nutricionales, culinarias y afrodisiacas (Cabanilla, 2010) estas últimas atribuidas de manera popular.

Los manglares y la pesquería de la concha prieta asociada a estos, tienen un valor fundamental en la dinámica social de las poblaciones locales, las cuales han obtenido de ellos su fuente de seguridad alimentaria y de sustento familiar (Guerrero, 2017).

Asimismo, el manglar y la concha prieta se han convertido en un referente social y cultural de las comunidades locales, alrededor de los cuales han articulado desde tiempos ancestrales su vida, su sentido de pertenencia y su identidad (Pincay-Pérez, 2012).

La extracción de la concha prieta genera beneficios para miles de familias recolectoras como su única fuente de ingresos económicos (Carchi et al., 2019), así como también a comerciantes, transportistas, cientos de cevicherías, picanterías, marisquerías y restaurantes que venden el producto en estado fresco y procesado (Cabanilla, 2010), en los famosos platos típicos como los ceviches, arroz con concha, arroz marinero, conchas asadas, el meloso de concha, concha en coco y otros que son apetecidos por turistas locales, nacionales y extranjeros (Maldonado, 2018).

Cabe señalar que la producción de concha prieta podría incrementarse al doble, pues tiene potencial de desarrollo con base en un manejo mejorado, técnicas de acuicultura de baja escala y bajo impacto ambiental, para así obtener los beneficios sociales, derivados de tal actividad (FAO, 2018).

Según Orquera (1999), la pesquería de los moluscos bivalvos y en general y por ende de la concha prieta, presenta múltiples ventajas y características que motivan a quienes participan en ella, principalmente a los concheros, a desarrollar esta actividad socioeconómica, entre las cuales destacan las siguientes:

- Fácil y segura ubicación: Ofrece poca o ninguna movilidad, por lo que hallar de nuevo sus localizaciones luego del reconocimiento inicial, es muy fácil y predecible (Moreno-Cáceres, 2014). 
- Producción relativamente constante: No presentan variaciones estacionales marcadas a nivel reproductivo ya que la gametogénesis es constante en estas especies, así como la cantidad de huevos viables desovados (FAO, 2007).

- Baja inversión y bajos riesgos de fracaso en su extracción: Su extracción manual plantea pocos riesgos debido a que requiere poco gasto de energía, no exige medios tecnológicos elaborados ni fuerza $\mathrm{O}$ adiestramiento especiales, además puede ser realizada por cualquier miembro de la familia incluido niños y ancianos y lo principal es que no requiere una inversión económica importante para realizar esta actividad (Orquera, 1999).

Es decir, la pesquería de la concha prieta es una actividad relativamente fácil de realizar, que genera ingresos constantes, con una baja inversión.

Otro factor que estimula su extracción es una demanda constante y creciente de la concha prieta como alimento en las cocinas de los hogares, cevicherías, picanterías y restaurantes del Ecuador y países vecinos, lo cual la hace llamativa a las poblaciones costeras de pescadores de bajos de recursos, he aquí la importancia social de la misma.

Sin embargo, esta actividad presenta desventajas entre la cuales destacan:

- Lento crecimiento o reposición del producto, con relación a la velocidad de la demanda existente: el tamaño de la concha depende de la velocidad de crecimiento (A. tuberculosa presenta un mayor crecimiento hasta los 0,9 años de edad alcanzando 39,5 mm LT y después de 1,1 años de edad con 44,7 mm LT su crecimiento es lento) Lucero et al. (2012); además está influenciado por condiciones ambientales (cantidad y disponibilidad del alimento; temperatura y salinidad del agua; densidad de población; ubicación en la zona interna real o por debajo de ella, etc.).

- Poca disponibilidad del recurso: por la alta presión predatoria principalmente de origen antrópico (Mora et al. 2010) producido por visitas frecuentes a los bancos donde crecen las conchas, por parte de más gente o recolecciones más intensivas, por lo que las conchas tendrían menos oportunidades de alcanzar su máximo desarrollo.

- Riegos laborales inherentes a su extracción (PradoCarpio et al., 2018b).

Estas desventajas podrían ser subsanadas a través de innovaciones y mejoras organizacionales, tecnológicas y ambientales, que se produzcan como resultado de procesos de investigación y desarrollo coordinados entre las universidades, las organizaciones de concheros y pescadores, el gobierno y la empresa privada, así como todos los interesados (Prado-Carpio et al., 2019)

Sin embargo, a pesar de todos los elementos antes destacados sobre la importancia de los aspectos sociales relacionados con la concha prieta, se debe señalar que, los concheros y otros participantes en la cadena de producción de la concha prieta, han experimentado una reducción de sus niveles de calidad de vida, cuyas familias según el BID (2017) tienen un ingreso de US\$ 4000/año/familia, muy por debajo de la canasta básica familiar en el Ecuador y además, altos niveles de pobreza que alcanzan en promedio un $83,5 \%$ por necesidades básicas insatisfechas (NBI).

Por otra parte, la actividad conchera presenta una gran problemática social subyacente, entre la cuales, según Beitl (2010), destacan: los conflictos entre los concheros y los camaroneros, entre los concheros asociados que cuentan con permisos para extraerla y otros concheros no asociados y no autorizados para extraer las conchas, así como también la inseguridad y la piratería.

\section{Importancia ambiental de la producción de la concha prieta}

La importancia ambiental de la concha prieta está estrechamente relacionada relacionada con la preservación y manejo del ecosistema (manglar), en tal virtud es necesario un ecosistema virtuoso, óptimo para que este molusco cumpla con éxito sus procesos biológicos.

Ahora bien, según Rodríguez et al. (2016), el ecosistema manglar en general crean múltiples beneficios que resaltan su importancia. A efectos de esta investigación se destacan los siguientes:

- Capturan y almacenan carbono atmosférico, produciendo el llamado carbono azul, el cual tiene incidencia global favorable para reducir el efecto invernadero y el calentamiento global (Mcleod et al., 2011)

- Conservan la biodiversidad, a través de servir de hábitat permanente o temporal para especies importantes, ya sea por ser endémicas, raras, amenazadas o en peligro de extinción (Félix-Pico et al., 2011)

- Mantienen las pesquerías locales, a través de la protección del hábitat de especies comerciales capturadas in-situ, tal es el caso de la de la concha prieta (Menéndez-Carrera y Guzmán, 2013).

- Mantienen las pesquerías costeras o de altura, a través de servir de refugio a especies comerciales durante sus etapas juveniles (León et al., 2001) 
- Proveen recursos no pesqueros, como madera para la construcción, carbón, leña, tanino y productos no maderables. Propiedades medicinales y uso apícola (Domínguez-Domínguez y Martínez-Zurimendi, 2019).

- Constituyen una franja de bosque protectora de las costas con función ecológica, económica y estratégico militar (PNUMA, 2015)

- Mantienen el equilibrio en la zona costera al impedir el avance de la intrusión salina (Bez-Collazo et al., 2019).

- Contienen la erosión costera por ser formadores de suelos (León et al., 2001).

- Reducen el riesgo de daños que puedan causar a la población, infraestructura productiva y cultivos agrícolas, eventos naturales como marejadas, tormentas tropicales y huracanes (CNA-SMN, 2005).

- Constituyen sitios de valores escénicos con importancia para el turismo ecológico (Arteaga-Cisnero et al., 2017).

- Evitan, al formar una red con las extensas ramificaciones de sus raíces, que la enorme carga de sedimento de los ríos llegue a mar abierto precipitadamente. Sin este filtro los fondos de algas marinas y los arrecifes se asfixian (Rodríguez et al., 2016).

- Proveen de sustancias nutritivas a muchas formas de vida no pueden encontrar alimento suficiente en los bancos de coral y en los fondos de algas marinas. Sin el abastecimiento de substancias orgánicas la abundancia de especies seguiría disminuyendo (Sanjurjo-Rivera y Welsh-Casas, 2005).

- Representan el hábitat en el cual alrededor de la mitad de todas las especies de peces se reproducen. Sin estas reservas, las colonias de peces disminuyen drásticamente. Aquí, junto a su valor ecológico, se hace patente también su valor económico (Hernández et al., 2019).

No obstante, las importantes funciones que tiene el ecosistema manglar, en Ecuador en los últimos 40 años se ha perdido más del $70 \%$ de las hectáreas (has) de manglar (Figura 3) debido al crecimiento urbano, al desarrollo de infraestructura turística y a la acuicultura (camaroneras). Sin embargo, se estima que aún existen más de 156 mil ha de manglar (BID, 2017).

Por lo tanto, una de las principales opciones históricas y de tipo técnico para la preservación y manejo del manglar lo representa la producción de la concha prieta, en conjunto con el cangrejo rojo (Ucides occidentalis) y otras especies del manglar, las cuales permiten su aprovechamiento económico y social con un mínimo impacto ambiental, si se realizan adecuadamente las labores productivas (Carchi et al., 2019).

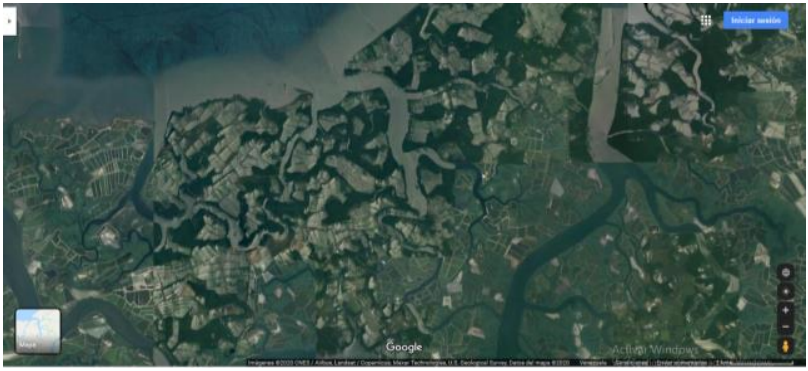

Figura 3 . Piscinas de camarones y área de manglar en Archipiélago de Jambelí

Fuente: Google Maps. 2020

En concordancia a lo establecido en la Ley Forestal del Ecuador, señala que los bosques de manglar son bienes nacionales y forman parte del Patrimonio Forestal del Estado, que solo pueden ser explotados mediante acuerdos de concesión comunitaria, pues el gobierno de Ecuador ha otorgado 40 concesiones a las comunidades tradicionales para su conservación (Márquez-Bustamante, 2014).

La economía de estas comunidades depende de la extracción/recolección de recursos marinos como el cangrejo rojo y la concha prieta.

El uso y aprovechamiento de recursos naturales que realizan las asociaciones de recolectores de los recursos del manglar es regulada por el Estado, a través de la Subsecretaría de Gestión Marino Costera (SGMC), con el objetivo de detener la deforestación en el país y promover la conservación de bosques y ecosistemas naturales, en los que se incluye a los manglares (Zambrano y Estupiñán, 2019).

Además, el gobierno creó el Programa Nacional de Incentivos a la Conservación y Uso Sostenible del Patrimonio Natural "Socio Bosque", como parte de este programa, desde el 2014, opera el Capítulo Socio Manglar (SM), el cual es pionero en América Latina y el Caribe (ALC) en incentivar económicamente a las comunidades para la conservación de los manglares (MAE, 2013).

Este modelo de concesiones ha logrado dar seguridad y certeza en el uso exclusivo del recurso y a promover una mejora en la productividad de algunas comunidades, no así, en generar emprendimientos exitosos (INABIO, 2019)

He aquí la importancia ambiental del desarrollo de un nuevo modelo de agronegocios para la producción de la concha prieta, lo cual redundará en la conservación del manglar, la producción, procesamiento y comercialización sostenible de la concha prieta y la mejora de las condiciones de vida de los concheros (Prado-Carpio et al, 2018), tal como ocurre con la producción de moluscos bivalvos en otras regiones del planeta.

\section{CONCLUSIONES}


- La producción de la concha prieta registra una relevancia significativa en términos económicos, sociales y ambientales para la producción ecuatoriana.

- En lo económico: su producción tiende a satisfacer el déficit del producto en el mercado ecuatoriano, reduciendo la salida de capitales al extranjero y contribuyendo así a la nivelación de la balanza comercial. Igualmente, su producción podría ser objeto de exportación en condiciones de óptima calidad y cumpliendo con todos los protocolos internacionales.

- En lo social: representa una significativa fuente de alimentación para las poblaciones vulnerables que habitan cerca de las costas aledañas a los manglares ecuatorianos.

- En lo ambiental: representa una actividad social y económica que se ha desarrollado históricamente en armonía y preservando el ecosistema del manglar, el cual se ha visto amenazado en su permanencia, por el desarrollo de diferentes actividades económicas y sociales, mismas que han incidido en la eliminación en grandes superficies del ecosistema manglar de la Costa ecuatoriana y el deterioro de sus condiciones naturales.

\section{LITERATURA CITADA}

Alcívar-Bravo, A. y Peñafiel-Rivera, T. 2018. Análisis Gastronómico de la concha prieta (Anadara Tuberculosa) y sus usos en la ciudad de Machala, sector Puerto Bolívar. Disponible en: http://repositorio.ug.edu.ec/handle/redug/35649.

Recuperado el 04.04.2020.

Alemán-Mejía, S.; Ordinola Zapata, E. y Montero, P. 2019. Concha negra Anadara tuberculosa (Sowerby, 1833) y concha huequera Anadara similis (C. B. Adams, 1852) en los manglares de Tumbes. Inf Inst Mar Perú 46(2):279-292.

Antón. B, Fierro. N. 2019. Pescadores Artesanales "Viquingos del mar". Primer Congreso Manglares de América. Universidad Espíritu Santo. Samborondón-Ecuador. Visible en: https://manglaresdeamerica.com/index.php/ec/arti cle/view/53/100. Recuperado el 21.05.2020.

Arias-Moran, A. E. y Castro Molina, K. V. 2017. Determinación de la influencia de los factores ambientales en el crecimiento y supervivencia de la concha prieta Anadara Tuberculosa. Trabajo final para la obtención del título: Ingeniero Oceánico Ambiental Espol FIMCBOR, Guayaquil. 106. Visible en: https://www.dspace.espol.edu.ec/handle/1234567 89/45353. Recuperado el: 21.05.2020.
Arteaga-Cisnero, J. P., Navarrete Pilacúa, M. A. Zambrano Camacho, N. R. 2017. La gestión del turismo ecológico y su impacto en la reserva ecológica Manglares Churute. Dominio de las Ciencias. 3(3):846-864.

Azabache Cobeña, J. 2016. "Cadena Productiva de (Anadara tuberculosa) (SOWERBY 1833) Extraida En El Santuario.” http://www.met.igp.gob.pe/publicaciones/2016/10T esis_Jose_Azabache.pdf. Recuperado el 21.0.2019.

BID (Banco Interamericano de Desarrollo). 2017. Competitividad de las comunidades concesionarias de manglares en el golfo de Guayaquil. Documento del Banco Interamericano de Desarrollo fondo multilateral de inversiones. $21 \mathrm{p}$.

Beitl, C. M. 2010. Situación Socio-Ecológica de la concha prieta (Anadara tuberculosa) y A. similis) en Ecuador y Recomendaciones. Informe Final Sobre los Resultados Preliminares de la Tesis Doctoral en Universidad de Georgia y Recomendaciones (Preinforme No. 1). Universidad de Georgia, Guayaquil-Ecuador. Visible en: https://www.academia.edu/5621453/Situaci\%C3 $\%$ B3n_Socio-

Ecol\%C3\%B3gica_de_la_Concha_Prieta_Anadar a_tuberculosa_y_A._similis_en_Ecuador_y_Reco mendaciones._Informe_Final_Sobre_los_Resultad os_Preliminares_de_la_Tesis_Doctoral_en_Unive rsidad_de_Georgia_y_Recomendaciones. Recuperado el 07.04.2020.

Benalcázar-Benavides, J. J. 2015. Estudio investigativo de la concha y su aplicación en la gastronomía. Universidad Tecnológica Equinoccial. Facultad de Turismo, Hotelería y Gastronomía. Carrera de Gastronomía. Tesis previa a la obtención del título de administrador gastronómico. $149 \mathrm{p}$. Visible en: http://repositorio.ute.edu.ec/xmlui/handle/1234567 89/16117. Recuperado el 04.04.2020.

Bez-Collazo, J; Rodríguez, L; González, A. 2019. Estudio exploratorio del agroecosistema costero de la cooperativa sabino pupo aledañas a Boca de Sama en Banes. Visible en: https://www.eumed.net/rev/oel/2019/10/agroecosist ema-costero-cooperativa.html. Recuperado el 06.04.2020.

Borda C.A. y Cruz R. 2004. Reproducción y reclutamiento del molusco (Anadara tuberculosa) (Sowerby, 1833) en el pacífico colombiano. Rev. Invest. Mar. 25(3):185-195. 
Cabanilla-Carpio, C. L. 2007. "Plan de Manejo de la Pesquería de concha prieta (Anadara tuberculosa) en la localidad de Puerto Hualtaco, Provincia de El Oro, Ecuador." Tesis de Maestría. Universidad Agraria del Ecuador. pg. 8.

Cabanilla, C. 2010. Comercialización de la concha prieta (Anadara tuberculosa) y A. símilis) en los principales puertos de la costa ecuatoriana. Visible en: https://www.oceandocs.org/handle/1834/4796. Recuperado el 04.04.2020.

Cáceres-Martínez, J. y Vásquez-Yeomans, R. 2014. Manual de Buenas Prácticas de Manejo para el Cultivo de Moluscos Bivalvos. Organismo Internacional Regional de Sanidad Agropecuaria (OIRSA) y Programa Regional de Apoyo a la Ejecución de la Política de la Pesca y la Acuicultura (PRAEPPESCA). 160 p.

Caicedo, J.L. 2014. Efectos sociales, económicos y ambientales en el proceso de extracción y comercialización del recurso (Anadara tuberculosa) (Sowerby 1833) en el cantón San Lorenzo, provincia de Esmeraldas. Visible en: http://dspace.utpl.edu.ec/handle/20.500.11962/218 25. Recuperado el 07.04.2020.

Calispa-Quinto, A. 2018. Cría en cautiverio de concha prieta (Anadara tuberculosa - Anadara similis) en el estuario del río Portoviejo y el estuario del río Chone para fortalecer los medios de vida tradicionales comunitarios, en los cantones Portoviejo, Sucre y San Vicente. Fundación FIDES. 26 p.

Camposano, J. A. 2020. El camarón alcanzó cifra récord en el 2019 en el Ecuador. Visible en: https:/www.elcomercio.com/actualidad/camaronrecord-ecuador-exportacion-economia.html. Recuperado el 04.04.2020.

Campos-Ocampo, M. 2017. Métodos de investigación académica. Fundamentos de investigación bibliográfica. $84 \mathrm{p}$.

Cañas-García, F. A. y Sierra O. S. 2019. Análisis bromatológico proximal en Anadara tuberculosa (Concha peluda) de la Bahía de Jiquilisco Departamento de Usulutan El Salvador. Bachelor thesis, Universidad de El Salvador. Visible en: http://ri.ues.edu.sv/id/eprint/20324/. Recuperado el 22.05.2020.

Carchi, X; Gaibor, N; Moreno, J; Alemán, C. y Ramírez, L. 2019. Los acuerdos de uso sostenible y custodia de manglar y su impacto en el recurso concha prieta (Anadara tuberculosa) en la provincia de Esmeraldas. Visible en: http:/www.manglaresdeamerica.com/index.php/e c/article/view/30/58. Recuperado el 05.04.2020.

CNA-SMN (Comisión Nacional del Agua - Servicio Meteorológico Nacional). 2005. Resumen del Huracán "Wilma" del océano atlántico. Servicio Meteorológico Nacional. Obtenido de http://smn.cna.gob.mx/tools/DATA/Ciclones\%20 Tropicales/Ciclones/2005-Wilma.pdf. Recuperado el 06.06.2020.

Cortes-Osorio, J. A. 2020. Una Revisión General de los Artículos de Revisión. Scientia et Technica Año XXV. 25(1): 2344-7214.

Cruz, R. A., Fonseca, C., and Chavarría-Solera, F. 2012. Comparación de La Composición Química Proximal de La Carne de (Anadara tuberculosa) y A. Similis (Bivalvia: Arcidae) de Chomes, Puntarenas. Rev. Mar. Cost. 4(1): 95-103.

Dazzini-Langdon, M. M. 2020. Metodología de planificación de escenarios para la resiliencia y la equidad de género: REMACAM, Ecuador. Revista Facultad de Comunicación, Lenguas y Literatura PUCE, 2020. Visible en: https://www.academia.edu/42189380/Metodolog $\% \mathrm{C} 3 \% \mathrm{ADa}$ de planificaci $\% \mathrm{C} 3 \% \mathrm{~B} 3 \mathrm{n}$ de escena rios_para_la_resiliencia_y_la_equidad_de_g\%C3 \%A9nero_REMACAM_Ecuador. Recuperado el $21.05 .202 \overline{0}$.

Dioses-Huayna, D. C. 2019. Caracterización del financiamiento y rentabilidad de las Mypes, sector comercio de recursos hidrobiológicos, concha negra (Anadara tuberculosa) provincia de Zarumilla, región Tumbes año 2018. Universidad Católica los Ángeles de Chimbote. Visible en: http://repositorio.uladech.edu.pe/handle/12345678 9/9994. Recuperado el 21.05.2020.

Diringer, B.; Pretell, K.; Avellan, R.; Chanta, C.; Cedeño, V. y Gentile, G. 2019. Genetic structure, phylogeography, and demography of Anadara tuberculosa (Bivalvia) from East Pacific as revealed by mtDNA: Implications to conservation. Ecology and Evolution, 9(8):4392-4402.

Domínguez-Domínguez, M. y P. Martínez-Zurimendi. 2019. Beneficios ambientales, usos forestales maderables y no maderables del manglar en la planicie costera. En: La biodiversidad en Tabasco. 
Estudio de Estado. 1. CONABIO, México, pp. 243247.

Espinosa G. S; Delgado, M.F.; Orobio, B.; MejíaLadino, L.M. y. Gil-Agudelo, D.L. 2010. Estado de la población y valoración de algunas estrategias de conservación del recurso piangua Andara tuberculosa (Sowerby) en sectores de Bazán y Nerete, costa pacífica nariñense de Colombia. Bol. Invest. Mar. Cost. 39 (1):161-176.

FAO (Food and Agriculture Organization of the United Nations). 2007. Estado actual del cultivo y manejo de moluscos bivalvos y su proyección futura. Factores que afectan su sustentabilidad en América Latina. Taller Técnico Regional de la FAO 20-24 de agosto de 2007, Puerto Montt, Chile. ISBN 97892-5-306115-0. Visible en: http://www.fao.org/3/i0444s/i0444s00.htm. Recuperado el 07.04.2020.

FAO (Food and Agriculture Organization of the United Nations). 2016. El estado mundial de la pesca y la acuicultura 2016. Contribución a la seguridad alimentaria y la nutrición para todos. Roma. 224 pp.

FAO (Food and Agriculture Organization of the United Nations). 2018. El estado mundial de la pesca y la acuicultura 2018. Cumplir los objetivos de desarrollo sostenible. Roma. Licencia: CC BYNC-SA $\quad 3.0 \quad$ IGO. Visible en: http://www.fao.org/publications/sofia/es/. Recuperado el 07.04.2020.

Farías, A. 2008. Nutrición y alimentación en moluscos bivalvos. En A. Lovatelli, A. Farías e I. Uriarte (eds). Estado actual del cultivo y manejo de moluscos bivalvos y su proyección futura: factores que afectan su sustentabilidad en América Latina. Taller Técnico Regional de la FAO. 20-24 de agosto de 2007, Puerto Montt, Chile. FAO Actas de Pesca y Acuicultura.No. 12. Roma, FAO. pp. 297-308.

Félix-Pico, E; Holguin-Quiñones, O y Escamilla-Montes, R. 2011. Macroinvertebrados marinos asociados al manglar. Departamento de Pesquerías y Biología Marina. Centro Interdisciplinario de Ciencias Marinas, Instituto Politécnico Nacional. Capítulo 8. pg 1. México.

Fundación de Defensa Ecológica. 2009. Acuerdo para precio de la concha prieta. Visible en: https://www.eluniverso.com/2009/03/19/1/1447/3
837B56A460B4B699F8762CF838D42D8.html. Recuperado el 04.04.2020.

Gómez, A. 2011. Género y desarrollo rural. En: El desarrollo rural en México y Colombia: problemas comunes y respuestas emergentes de los actores coordinadores. 1a ed. -- Bogotá : Editorial Pontificia Universidad Javeriana ; [Coyoacán, México, D.F.]: Universidad Autónoma Metropolitana, Unidad Xochimilco. Pág. 147 a $150.312 \mathrm{p}$.

Gómez-Luna, E., Fernando-Navas, D., Aponte-Mayor, G., Betancourt-Buitrago, L. A. 2014. Metodología para la revisión bibliográfica y la gestión de información de temas científicos, a través de su estructuración y sistematización. Dyna. 81(184): 158-163.

González-Banchón, T. y Solano-Vera, Y. 2019. Estudio de la biometría de anadara tuberculosa (concha prieta) comercializada en el mercado. Journal of business and entrepreneurial studies. 3(1): 27-31.

Google Maps. 2020. Imagen Archipiélago Jambelí, Provincia de El Oro, Ecuador. Visible en: https:/www.google.es/maps/search/archipielago+de +jambel\%C3\%AD+ecuador/@-3.3595864,-

$80.0931822,11757 \mathrm{~m} /$ data $=! 3 \mathrm{~m} 1$ ! $1 \mathrm{e} 3$. Recuperado el 06.04.2020.

Guerrero, P. 2017. Manglar y pesquerías: Seguridad alimentaria y reducción de pobreza. Visible en: https:/www.wwf.org.ec/?307590/Cangrejos-enmercado-de-Caraguay. Recuperado el 05.04.2020.

Helm, M.M.; Bourne, N.; Lovatelli, A. 2006. Cultivo de bivalvos en criadero. Un manual práctico. FAO Documento Técnico de Pesca. No. 471. (comp./ed.), Roma, FAO. 2006. 184 pp. Visible en: http://www.fao.org/3/y5720s/y5720s04.htm\#bm0 4. Recuperado el 07.04.2020.

Hernández C. L.; Fox Cuthbert, K. y Rivas S. E. 2019. Afectaciones antropogénicas al ecosistema manglar en la zona costera de la comunidad de Pearl, Lagon, municipio Laguna de Perlas. Revista Universitaria del Caribe, 22(1).

IAES (Instituto de Acuacultura del Estado de Sonora). S/F. Moluscos bivalvos. Visible en: https://www.iaes.gob.mx/index.php?pag=producto s. Recuperado el 27.02.2020.

INABIO (Instituto Nacional de Biodiversidad). 2019. Desarrollo sostenible de las comunidades ancestrales del ecosistema de manglar. Visible en: 
http://inabio.biodiversidad.gob.ec/2019/01/31/40desarrollo-sostenible-de-las-comunidadesancestrales-del-ecosistema-de-manglar/. Recuperado el 06.04.2020.

Ipac 2017. El comercio internacional de bivalvos no alcanza el 5\% del total producido. Visible en: http://www.ipacuicultura.com/noticias/en_portada /54616/el_comercio_internacional_de_bivalvos_n o_alcanza_el_5_del_total_producido.html. Recuperado el 07.04.2020.

Lazarich-Gener, R. 2009. Estudio de mercado de la concha negra (Anadara similis y Anadara tuberculosa) en Nicaragua. Comercialización con garantía de inocuidad. Centro de Investigación de Ecosistemas Acuáticos - CIDEA de la Universidad Centroamericana. $55 \mathrm{p}$.

Lee, R.; Lovatelli, A.; Ababouch, L. 2010. Depuración de bivalvos: aspectos fundamentales y prácticos. FAO Documento Técnico de Pesca. No. 511. Roma, FAO. 2010. 153pp.

León de la Luz, J. L; Félix-Pico, E. F; RiosmenaRodríguez, R. y Serviere-Zaragoza, E. 2011. Los manglares de la península de baja california. Centro Interdisciplinario de Ciencias Marinas, Centro de Investigaciones Biológicas del Noroeste, S.C. y Universidad Autónoma de Baja California Sur.pg 10. México.

Lovatelli, A., Vannuccini, S. y McLeod, D. 2008. Current status of world bivalve aquaculture and trade. En A. Lovatelli, A. Farías e I. Uriarte (eds). Estado actual del cultivo y manejo de moluscos bivalvos y su proyección futura: factores que afectan su sustentabilidad en América Latina. Taller Técnico Regional de la FAO. 20-24 de agosto de 2007, Puerto Montt, Chile. FAO Actas de Pesca y Acuicultura. No. 12. Roma, FAO. pp. 45-59.

Lucero, C., J. Cantera y R. Neira. 2012. "Pesquería y crecimiento de la piangua (Arcoida: Arci dae) (Anadara tuberculosa) en la Bahía de Málaga del Pacífico colombiano, 2005-2007.” Rev. Biol. Trop. 60(1): 203-217.

Luchini, L. y Panné Huidobro, S. 2008. Perspectivas en acuicultura: nivel mundial, regional y local. Secretaria de agricultura, ganadería, pesca y alimentos del Gobierno de Argentina. 99 p.

MAE (Ministerio del Ambiente de Ecuador). 2013. Acuerdo Ministerial N¹31: Programa Nacional de Incentivos a la Conservación y Uso Sostenible del
Patrimonio Natural "Socio Bosque". Visible en: https://ecuadorforestal.org/legislacionvigente/7935/. Recuperado el 06.04.2020.

MAGAP (Ministerio de Agricultura y Ganadería de Ecuador). 2014. Ecuador y Colombia analizan el estado de la concha prieta. Visible en: https://www.agricultura.gob.ec/ecuador-ycolombia-analizan-el-estado-de-la-conchaprieta/\#. Recuperado el 20.02.2020.

Maldonado, J. E. 2018. Aplicación de la concha prieta ecuatoriana en la alta cocina. Proyecto de titulación. Facultad de Turismo, Hospitalidad y Gastronomía. 67 p. Visible en: http://dspace.udla.edu.ec/handle/33000/8620. Recuperado el: 08.05.2019.

Márquez-Bustamante, C. 2014. Plan de manejo de los manglares del estero Guajabal en el archipiélago de Jambelí. Visible en: http://repositorio.utmachala.edu.ec/handle/48000/ 1981. Recuperado el 06.04.2020.

Mcleod, E., Chumra, G., Bouillon, S., Salm, R., Bjork, M., Duarte, C., Lovelock, C., Schlesinger, W. y Siliman, B. 2011. A blueprint for blue carbon: toward an improved understanding of the role of vegetated coastal habitats in sequestering $\mathrm{CO} 2$. Frontiers in Ecology and the Environment 9(10): $552-560$.

Mendoza N, O. y Alvitres, V. 2013. Crecimiento y supervivencia de Anadara tuberculosa (Sowerby, 1833) a tres densidades. Universidad Nacional de Tumbes, Perú, Revista de Investigación Científica Manglar 12(1): 55-64.

Mendoza, J. 2015. "Evaluación de La Pesquería de La concha prieta (Anadara Tuberculosa) y de La Concha Mica (Anadara Similis) En La Provincia de El Oro, Ecuador. Universidad Técnica de Machala. $117 \mathrm{p}$.

Mendoza-Portillo F, Ramírez-Rodríguez M, VargasLópez V. 2020. Interactions of small-scale fisheries in Mexico's northwest Pacific. Lat. Am. J. Aquat. Res. 48(1): 94-105.

Menéndez-Carrera, L y Guzmán, J. 2013. Ecosistemas de manglar en el archipiélago cubano. Editorial Academia, Cuba. 471 p. Visible en: https://unesdoc.unesco.org/ark:/48223/pf0000178 568. Recuperado el 06.04.2020. 
Molar-Orozco, M. E. 2020. Métodos y técnicas de investigación. $56 \mathrm{p}$.

Mora, E. y Moreno, J. 2009. La pesquería artesanal del recurso concha (Anadara tuberculosa y A. similis) en la costa ecuatoriana durante el 2004. Boletín Científico y Técnico. 20(1):1-16.

Mora, E; Moreno, J; Jurado, V. y Flores, L. 2010. La Pesquería de La concha prieta (Anadara tuberculosa) y Anadara Similis) en el 2009: Indicadores Pesqueros y Condición Reproductiva En La Zona Sur y Norte de Ecuador.” Boletín Científico y Técnico 20(8): 35-49.

Moreno-Cáceres, J. 2014. Situación pesquera del recurso concha prieta en los principales puertos de la provincia Esmeraldas durante el 2013. Instituto de Pesca de Ecuador. 16 p.

Morocho, V.; Morales, P.; Escalante, S.; De la Cruz,. J,; Morales, S.; Morales, L.; Vasconez, O.; Vera, I.; Medina, J.; Terán, C.; Carvajal, R.; Chalen, X. 2019. Mancomunidad de organizaciones pesqueras de puerto del morro para la conservación de los manglares y sus recursos pesqueros. Manglares de Ecuador. Visible en: https://manglaresdeamerica.com/index.php/ec/arti cle/view/45/90. Recuperado el: 21.05.2020.

Muñoz-Suarez, C. 2018. Diagnostico socioeconómico del agronegocio conchero (Anadara tuberculosa) en la comuna Las Huacas del Archipiélago de Jambelí. Universidad Técnica de Machala, Ecuador. Trabajo titulación. 66 p.

Murillo-Rivas, J. 2018. Evaluación del efecto de la temperatura en el desove de la piangua Anadara tuberculosa (Bivalva: Arcidae) en condiciones de laboratorio. Universidad del Magdalena. Maestría en Acuicultura. Trabajo de grado. Visible en: http://repositorio.unimagdalena.edu.co/jspui/handl e/123456789/3145. Recuperado el: 21.05.2020.

Observatorio de la Complejidad Económica. 2018. Productos. Moluscos. Visible en: https://atlas.media.mit.edu/es/profile/hs92/0307/. Recuperado el 07.04.2020.

Ordinola, E; Alemán, M, S; Inga-Barreto, C; Vera, M. y Llanos, J. 2019. Sinopsis biológica, poblacional y pesquera de Anadara tuberculosa (Sowerby, 1833) y Anadara similis (C.B. Adams, 1852) en los manglares de Tumbes: 1995 a 2015. Bol Inst Mar. 34(1):223-264.
Orquera, L 1999. El Consumo de moluscos por los canoeros del extremo sur. Relaciones de la Sociedad Argentina de Antropología XXIV. ISSN 0325-2221. Buenos Aires, 21 p.

Ortiz-Bolaños, D. C. 2019. Análisis de la cadena de valor de la concha negra (Anadara tuberculosa) para el uso sostenible en áreas concesionadas de las comunidades Bunche y Sálima a usuarios/as del ecosistema manglar del cantòn Muisne, provincia de Esmeraldas. (Tesis de grado. Ingeniero Forestal). Escuela Superior Politécnica de Chimborazo. Riobamba. Visible en: http://dspace.espoch.edu.ec/handle/123456789/12 407. Recuperado el: 21.05.2020.

Pacheco-Navarro, C. G. 2017. Etnoarqueología de la actividadconchera en la isla puná. Trabajo final para la obtención del título: Magister en Arqueología del Neotrópico. Espol. FICT., Guayaquil. Visible en: https://www.dspace.espol.edu.ec/handle/1234567 89/39729. Recuperado el: 21.05.2020.

Panta-Vélez, R. P.; Bermúdez-Medranda, A.; Mero, P.; Arrieche, D. y Acosta-Balbás, Vanessa. 2020. Reproductive Cycle of Anadara tuberculosa (Sowerby, 1833) (Bivalvia: Arcidae) in a Mangrove System of the Chone River Estuary, Ecuador. Advances in Environmental Biology, 14(2):1-11.

Pincay-Pérez, B. 2012. Estructura administrativaoperativa comunitaria para la gestión de los emprendimientos eco-turísticos del manglar de palmar en el periodo 2012. Visible en: https://repositorio.upse.edu.ec/xmlui/handle/4600 0/760. Recuperado el 05.04.2020.

Pintó-Solé, R. M.; Martín de Santos, M. y Bosch-Navarro, A. 2011. Informe del Comité Científico de la Agencia Española de Seguridad Alimentaria y Nutrición (AESAN) sobre contaminación vírica de los alimentos, con especial énfasis en moluscos bivalvos, y métodos de control. Revista del comité científico. 14:89-105.

PNUMA (Programa de las Naciones Unidas para el Medio Ambiente). 2015. Medidas para la gestión ecosistémica de las zonas marinas y costeras-Guía de Introducción. ISBN: 978-92-807-3173-6.

Prado-Carpio, E., Martínez-Soto, M., Urdaneta de Galué, F., Morris-Díaz, A. T. y Rodríguez-Monroy, C., Borja-Herrera, A. 2018b. Modelo Teórico de Relaciones entre la Gestión de Agronegocios y el 
Desempeño de la Cadena de Valor de la concha prieta (Anadara tuberculosa). Visible en: http://www.laccei.org/LACCEI2018-

Lima/meta/FP396.html. Recuperado el 04.04.2020.

Prado-Carpio, E., Quezada-Abad, C., Martínez-Soto, M., Rodríguez-Monroy, C., y Morris-Díaz, 2018a. An Approximation to Agribusiness Development in the Value Chain of the Bivalve Mollusk « (Anadara tuberculosa) (Sowerby, 1833) (Arcidae)». Proceedings in Food System Dynamics, 382393.http://centmapress.ilb.unibonn.de/ojs/index.php/proceedings/article/view/18 $\underline{31}$.

Prado-Carpio, E; Castro-Armijos, C; Rentería-Minuche, P; Coronel-Reyes, J; Paladines-Amaiquema, J y Martínez-Soto, M. 2019. Agronegocio Conchero (Anadara tuberculosa): Aspectos socioeconómicos, Archipiélago de Jambelí. Revista Cumbres. 5(2):79-88.

Rendón-Yllescas, M. K., Suárez Gómez, E. V. y M.T. Mejía. 2009. Manejo Sustentable Y Comercialización De concha prieta En Cautiverio, En Puerto El Morro--Provincia Del Guayas, http://www.dspace.espol.edu.ec/xmlui/handle/123 456789/1426?show=full. Recuperado el 07.04.2020.

Rivas-Mejía, J. R. 2018. Determinación de minerales y metales pesados en Anadara tuberculosa (Concha peluda) en la Bahía de Jiquilisco Doartamento de Usulután El Salvador. Bachelor thesis, https://ues.edu.sv. Recuperado 22.05.2020.

Rodríguez Crespo, G., Chiriboga Calderón, F., y Lojan Feijoo, A. 2016. las camaroneras ecuatorianas: una polémica medioambiental. Revista Universidad y Sociedad, 8(3): 151-156.

Sanjurjo-Rivera, E; Welsh-Casas, S. 2005. Una descripción del valor de los bienes y servicios ambientales prestados por los manglares Gaceta Ecológica, núm. 74, enero-marzo, 2005, pp. 55-68. Secretaría de Medio Ambiente y Recursos Naturales Distrito Federal, México.

Silva-Benavides, A y Bonilla, R. 2014. Estructura de la población y distribución de Anadara tuberculosa Sowerby (1833) (Mollusca: Bivalvia) en los manglares de Golfito y Playa Blanca de Puerto Jiménez, Golfo Dulce, Costa Rica. Rev. Biol. Trop. 63(1): 287-298.
Tapia-Santos, D. C. 2020. Picantería Don Viche en la ciudad de Babahoyo. Universidad Técnica de Babahoyo. Visible en: http://dspace.utb.edu.ec/handle/49000/7434. Recuperado el: 21.05.2020.

Tripaldi, P.; Carrasco Peña, M. y Webster Coello, G. R. 2016. Capacidad bioacumuladora de metales pesados en moluscos bivalvos de los esteros del cantón Balao. Universidad del Azuay. Visible en: http://dspace.uazuay.edu.ec/handle/datos/6480. Recuperado el 22.05.2020.

Valencia-Caicedo, K. 2013. Factores determinantes de la competitividad del sector conchero en comunidades de la reserva ecológica manglares cayapas mataje (remacam), del cantón San Lorenzo, Esmeraldas: caso de estudio FEDARPOM [Internet]. Facultad Latinoamerica de Ciencias Sociales FLACSO; 2013. Disponible en:

https://repositorio.flacsoandes.edu.ec/xmlui/handl e/10469/6960. Recuperado el 04.04.2020.

Wijsman J.W.M., Troost K., Fang J., Roncarati A. 2019. Global Production of Marine Bivalves. Trends and Challenges. In: Smaal A., Ferreira J., Grant J., Petersen J., Strand Ø. (eds) Goods and Services of Marine Bivalves. Springer, Cham

Zambrano, L y Estupiñán, R. 2019. Asociación de pescadores y recolectores de productos del mar ASOPESCMAR: Experiencias con el acuerdo de uso sustentable y custodia de manglar 2016 - 2019. Manglares de Ecuador Páginas 271 a 279. Visible en:

http:/www.manglaresdeamerica.com/index.php/e c/article/view/43/86. Recuperado el 06.04.2020.

Zavala-Arellano, J. M. 2019. Aislamiento y caracterización molecular de bacterias oxidadoras de azufre presentes en la sangre del molusco bivalvo Anadara tuberculosa (Sowerby 1833) “concha negra". Universidad Nacional Pedro Ruiz Gallo. http://190.108.84.117/handle/UNPRG/4771 Recuperado el 21.05.2020. 